PROCEEDINGS OF THE

AMERICAN MATHEMATICAL SOCIETY

Volume 135, Number 12, December 2007, Pages 3967-3975

S 0002-9939(07)09007-7

Article electronically published on August 1, 2007

\title{
A REPRODUCING KERNEL CONDITION FOR INDETERMINACY \\ IN THE MULTIDIMENSIONAL MOMENT PROBLEM
}

\author{
ROGER A. ROYBAL
}

(Communicated by Joseph A. Ball)

\begin{abstract}
Using the smallest eigenvalues of Hankel forms associated with a multidimensional moment problem, we establish a condition equivalent to the existence of a reproducing kernel. This result is a multivariate analogue of Berg, Chen, and Ismail's 2002 result. We also present a class of measures for which the existence of a reproducing kernel implies indeterminacy.
\end{abstract}

\section{INTRODUCTION}

In 2], Berg, Chen, and Ismail find a new condition equivalent to determinacy in the one-dimensional moment problem.

Theorem 1.1 (Berg, Chen, and Ismail, 2002). Let $\lambda_{N}$ be the smallest eigenvalue of the truncated Hankel matrix $H_{N}$ for the measure $\mu$. Then $\lambda_{N} \rightarrow 0$ as $N \rightarrow \infty$ if and only if $\mu$ is determinate.

They use the classical fact that a measure is indeterminate if and only if a reproducing kernel exists on the Hilbert space in which the polynomials are dense. Additionally, a reproducing kernel exists if and only if the sum

$$
\sum_{k=0}^{\infty}\left|P_{k}\left(z_{0}\right)\right|^{2}
$$

converges for some $z_{0} \in \mathbb{C} \backslash \mathbb{R}$, where $\left\{P_{k}\right\}$ is the standard set of orthonormal polynomials. These results go back to M. Riesz and may be found in [1].

Let $\mu$ be a positive measure such that $s_{n}=\int_{\mathbb{R}} x^{n} d \mu$ is finite for all $n$. For each $N \in \mathbb{N}$, we define the $N^{\text {th }}$ Hankel matrix to be

$$
H_{N}=\left(s_{i+j}\right)_{i, j=0}^{N} .
$$

Since $\mu$ is positive, this implies that $H_{N}$ is positive semidefinite for each $N$. We let $\lambda_{N}$ be the smallest eigenvalue of $H_{N}$ and note that Cauchy's interlace theorem implies that $\lambda_{N}$ decreases as $N$ increases. If $\lambda_{N}=0$ for some $N$, then $\lambda_{n}=0$ for

Received by the editors May 30, 2006 and, in revised form, November 11, 2006

2000 Mathematics Subject Classification. Primary 47A57; Secondary 46E22.

Key words and phrases. Multidimensional moment problem, reproducing kernel, Hankel matrix.

The author would like to thank Mihai Putinar for all his advice and support during the preparation of this article. 
all $n \geq N$, and the measure is a finite sum of point masses [1. This case implies determinacy since any measure with compact support is automatically determinate. In the case where $\mu$ has infinite support, then $\lambda_{N}>0$ for every $N$.

Berg, Chen, and Ismail show that $\lambda_{N} \rightarrow \gamma>0$ if and only if a reproducing kernel exists for the polynomials, which gives the conclusion. We propose to extend this to a similar question in the multidimensional setting.

Let $\alpha=\left(\alpha_{1}, \alpha_{2}, \ldots, \alpha_{d}\right) \in \mathbb{N}_{0}^{d}$ be a multi-index, $x=\left(x_{1}, x_{2}, \ldots, x_{d}\right) \in \mathbb{R}^{d}$, and let $x^{\alpha}=x_{1}^{\alpha_{1}} x_{2}^{\alpha_{2}} \cdots x_{d}^{\alpha_{d}}$. We shall use the standard notation of $|\alpha|=\alpha_{1}+\cdots+$ $\alpha_{d}$. For a given multisequence $\left\{s_{\alpha}\right\}$, define a linear functional $L: \mathbb{C}[x] \rightarrow \mathbb{C}$ by $L\left(x^{\alpha}\right)=s_{\alpha}$, and extend linearly. We say the multisequence $\left\{s_{\alpha}\right\}$ and the associated functional $L$ are positive if for every polynomial $p \in \mathbb{C}[x]$, we have $L(p \bar{p}) \geq 0$. When $L$ is positive, we construct a pre-inner product by $\langle p, q\rangle=L(p \bar{q})$. If we consider the ideal $\mathcal{N}=\{p \in \mathbb{C}[x]:\langle p, p\rangle=0\}$, then $\langle\cdot, \cdot\rangle$ acting on $\mathbb{C}[x] / \mathcal{N}$ becomes a postive definite form, and we complete this to a Hilbert space $\mathcal{P}$ in which $\mathbb{C}[x] / \mathcal{N}$ is dense. This is the standard GNS construction and may be found in full detail in [5]. We will also assume a normalizing condition of $s_{(0,0, \ldots, 0)}=1$ meaning that we deal with probability measures. This assumption also simplifies many calculations.

We define the Hankel kernel indexed over $\alpha$ associated with the multisequence $\left\{s_{\alpha}\right\}$ by

$$
H=\left(s_{\alpha+\beta}\right)_{\alpha, \beta \in \mathbb{N}_{0}^{d}}
$$

and for any $N \in \mathbb{N}$ the $N^{\text {th }}$ truncation of $H$ by

$$
H_{N}=\left(s_{\alpha+\beta}\right)_{0 \leq|\alpha|,|\beta| \leq N}
$$

One significant difference between the multidimensional and one dimensional cases is that in one variable, a positive measure $\mu$ exists in $\mathbb{R}$ which represents $L$ in the sense that $L(p)=\int_{\mathbb{R}} p d \mu$ if and only if $L$ is positive, whereas there exist positive $L$ for which there are no such $\mu$ on $\mathbb{R}^{d}$ if $d \geq 2$, examples of which were concurrently discovered in [3] and [9]. In the case where such a $\mu$ exists, its support must lie in the real algebraic variety generated by the ideal $\mathcal{N}$. If we denote by $\lambda_{N}$ the smallest eigenvalue of the matrix $H_{N}$, then $\lambda_{N} \geq 0$ for all $N \in \mathbb{N}$ and equality holds for some $N$ if and only if $\mathcal{N}$ is a nontrivial ideal of $\mathbb{C}[x]$. For the remainder of this article we will assume that $\mathcal{N}=(0)$ so that $\lambda_{N}>0$ for all $N$.

If we try to bring Berg, Chen, and Ismail's result to the multivariate case we must find a new statement since there exist indeterminate measures $\mu$ for which $\lambda_{N} \rightarrow 0$ as $N \rightarrow \infty$.

To show this we use a theorem of Petersen [6].

Theorem 1.2 (L. C. Petersen). Let $\mu$ be a moment measure on $\mathbb{R}^{d}$. Consider the projection $\pi_{j}: \mathbb{R}^{d} \rightarrow \mathbb{R}$ onto the $j^{\text {th }}$ coordinate and the projection measures $\mu_{j}:=\pi_{j *}(\mu)$ on $\mathbb{R}$.

(a) If each $\mu_{j}$ is determinate, then so is $\mu$.

(b) If we assume that $\mu=\mu_{1} \otimes \mu_{2} \otimes \cdots \otimes \mu_{d}$, then $\mu$ is determinate if and only if $\mu_{j}$ is determinate for $1 \leq j \leq d$.

Example 1.3. Let $\mu_{1}$ and $\mu_{2}$ be measures on $\mathbb{R}$ admitting all moments with $\mu_{1}$ determinate and $\mu_{2}$ indeterminate. Let $\nu$ be a measure on $\mathbb{R}$ distinct from $\mu_{2}$ but possessing the same moments. Define $\mu:=\mu_{1} \otimes \mu_{2}$. Clearly $\mu$ is indeterminate since $\mu_{1} \otimes \nu$ gives the same moments as $\mu$. We now show that for $\mu, \lambda_{N} \rightarrow 0$ as $N \rightarrow \infty$. 
Consider the matrix $J_{N}=\left(s_{(m+n, 0)}\right)_{0 \leq m, n \leq N}$, and let $\eta_{N}$ be its smallest eigenvalue. Then $J_{N}$ is a principal submatrix of the matrix $H_{N}$, and by their selfadjointness and Cauchy's interlace theorem we see that $0 \leq \lambda_{N} \leq \eta_{N}$. Note that $J_{N}$ is the $N^{\text {th }}$ Hankel matrix associated with the measure $\mu_{1}$ which is determinate in $\mathbb{R}$, whence $\eta_{N} \rightarrow 0$ as $N \rightarrow \infty$ by [2]. Thus $\mu$ is an indeterminate measure such that $\lambda_{N} \rightarrow 0$.

A reproducing kernel for $\mathcal{P}$, the completion of the polynomials on $\mathbb{C}^{d}$, is a function $K: \mathbb{C}^{d} \times \mathbb{C}^{d} \rightarrow \mathbb{C}$ which has the reproducing property, i.e. so that for any polynomial $p(x)$,

$$
p(y)=\langle p(x), K(x, y)\rangle_{\mathcal{P}} .
$$

We construct a complete system of orthonormal polynomials $\left\{P_{\alpha}\right\}_{\alpha \in \mathbb{N}_{0}^{d}}$ which serve as an orthonormal basis of $\mathcal{P}$. Typically one works with orthonormal polynomials in which $\operatorname{deg}\left(P_{\alpha}\right)$ is an increasing with $|\alpha|$, and here we assume that $\operatorname{deg}\left(P_{\alpha}\right)=$ $|\alpha|$. This may be achieved by ordering $\left\{x^{\alpha}\right\}_{\alpha \in \mathbb{N}_{0}^{d}}$ in a graded lexicographical order and then using the Gram-Schmidt procedure to obtain an orthonormal system of polynomials. The construction of orthonormal polynomials from the monomials may be found in [4].

Using this basis of orthonormal polynomials, we find

$$
K(x, y)=\sum_{\alpha \in \mathbb{N}_{0}^{d}} P_{\alpha}(x) \overline{P_{\alpha}(y)}
$$

and for a fixed $y \in \mathbb{C}^{d}$, this is a function in $\mathcal{P}$ provided

$$
\sum_{\alpha \in \mathbb{N}_{0}^{d}}\left|P_{\alpha}(y)\right|^{2}
$$

is finite. It follows that if this sum is finite, point evaluation at $y$ is a bounded linear functional in $\mathcal{P}$, i.e. there is a constant $C_{y}$ so that $|p(y)|^{2} \leq C_{y} L(p \bar{p})$. Riesz's theorem provides representation of this functional by the element $K(x, y)$ as a function in the variable $x$.

Our focus will be on the sum (1.1) to show that it converges for every $y \in \mathbb{C}^{d}$. In particular we will want this sum to be uniformly bounded on compact subsets of $\mathbb{C}^{d}$. More information on reproducing kernels may be found in $[$ ].

\section{MAin RESUlt}

For $R>0$ define the $R$-scaling of the multisequence $\left\{s_{\alpha}\right\}$ to be $\left\{\frac{s_{\alpha}}{\left.R^{|\alpha|}\right\}}\right.$ and the associated truncated Hankel matrices from this $R$-scaling:

$$
H_{R, N}=\left(\frac{s_{\alpha+\beta}}{R^{|\alpha+\beta|}}\right)_{0 \leq|\alpha|,|\beta| \leq N} .
$$

Note that if $\left\{s_{\alpha}\right\}$ is the moment multisequence of a measure $\sigma$, then the $R$-scaling of $s$ is the moment multisequence of the measure $\sigma_{R}$, where $\sigma_{R}(B)=\sigma(R B)$ for every Borel set $B$; we define $R B=\{R b: b \in B\}$ in the natural way and define the $R$-scaling of the measure $\sigma$ to be $\sigma_{R}$. In the following theorem, we only assume that $\left\{s_{\alpha}\right\}$ is a positive multisequence, not necessarily that it is a moment multisequence as we do in the one dimensional case.

We define $\mathcal{P}_{R}$ to be the Hilbert space associated with the $R$-scaling of $\left\{s_{\alpha}\right\}$. If $f(x)$ is in the Hilbert space $\mathcal{P}$ associated with the sequence $s_{\alpha}$, then the mapping 
$\phi_{R}: \mathcal{P} \rightarrow \mathcal{P}_{R}$ given by $\phi_{R}(f)(x)=f(R x)$ is the required isometry. We will sometimes denote $\phi_{R}(f)$ as $f_{R}$.

Using this isometry, we also relate a system of orthonormal polynomials of the original form to a system of the scaled form by letting $P_{R, \alpha}(z)=P_{\alpha}(R z)$. We choose a set of orthonormal polynomials so that $\operatorname{deg}\left(P_{\alpha}\right)=\operatorname{deg}\left(P_{R, \alpha}\right)=|\alpha|$ (see [4]).

Let $\lambda_{R, N}$ be the smallest eigenvalue of $H_{R, N}$, and let $\lambda_{R, N} \rightarrow \gamma_{R}$ as $N \rightarrow \infty$. We now state the main result.

Theorem 2.1. Assume that $\lambda_{R, N}>0$ for all $N \in \mathbb{N}$ and some $R>0$. A reproducing kernel for the polynomials exists which is uniformly bounded on compact sets if and only if $\gamma_{R}>0$ for every $R>0$.

Proof. This proof follows closely that of Berg, Chen, and Ismail. The primary difference is in the use of scalings of a multisequence. This is from the fact that it is an open problem as to whether in the multivariate case the sum $\sum_{\alpha}\left|P_{\alpha}(z)\right|^{2}$ converging at some nonreal $z$ implies the existence of a reproducing kernel as it does in the one variable case.

We begin by writing the smallest eigenvalue of $H_{R, N}$ as the Rayleigh quotient

$$
\lambda_{R, N}=\min \left\{\sum_{|\alpha| \leq N} \sum_{|\beta| \leq N} \frac{s_{\alpha+\beta}}{R^{|\alpha+\beta|}} v_{\alpha} \overline{v_{\beta}}: \sum_{|\alpha| \leq N}\left|v_{\alpha}\right|^{2}=1\right\} .
$$

Note that if $\lambda_{R, N}>0$ for some $R>0$ and $N \in \mathbb{N}$, then $\lambda_{S, N}>0$ for any $S>0$. We show this by defining $L_{R}$ to be the functional derived from the multisequence $\left\{\frac{s_{\alpha}}{R^{|\alpha|}}\right\}$; then for any polynomial $p(x), L_{R}(p(x))=L_{S}\left(p\left(\frac{S x}{R}\right)\right)$. We also write for $p(x)=\sum_{|\alpha| \leq N} a_{\alpha} x^{\alpha}$,

$$
\lambda_{R, N}=\min \left\{L_{R}\left(|p|^{2}\right): \sum_{|\alpha| \leq N}\left|a_{\alpha}\right|^{2}=1, \operatorname{deg}(p) \leq N\right\} .
$$

Therefore if $L_{R}\left(|p|^{2}\right)=0$ for some polynomial $p$, then we conclude that $\lambda_{S, N}=0$ for any $S>0$.

We note that the monomials are orthonormal with respect to normalized Lebesgue measure on the unit torus, so we rewrite the smallest eigenvalue as

$$
\lambda_{R, N}=\min \left\{L_{R}\left(|p|^{2}\right): \int_{\theta \in[0,2 \pi]^{d}}\left|p\left(e^{i \theta}\right)\right|^{2} \frac{d \theta}{(2 \pi)^{d}}=1, \operatorname{deg}(p) \leq N\right\} .
$$

Since $\lambda_{R, N}>0$ we write

$$
\frac{1}{\lambda_{R, N}}=\max \left\{\int_{\theta \in[0,2 \pi]^{d}}\left|p\left(e^{i \theta}\right)\right|^{2} \frac{d \theta}{(2 \pi)^{d}}: L_{R}\left(|p|^{2}\right)=1, \operatorname{deg}(p) \leq N\right\} .
$$

We rewrite the polynomial $p(x)=\sum_{|\alpha| \leq N} c_{\alpha} P_{R, \alpha}(x)$, where $\left\{P_{R, \alpha}\right\}$ are the standard orthonormal polynomials with respect to a fixed ordering in which $m \geq n \Rightarrow$ $\left|\alpha_{m}\right| \geq\left|\alpha_{n}\right|$ for $m, n \in \mathbb{N}$ (see [4]). Now considering the matrix $\mathcal{K}_{R}$, where

$$
\mathcal{K}_{R, \alpha, \beta}=\int_{\theta \in[0,2 \pi]^{d}} P_{R, \alpha}\left(e^{i \theta}\right) \overline{P_{R, \beta}\left(e^{i \theta}\right)} \frac{d \theta}{(2 \pi)^{d}} .
$$


We express the equation above as another eigenvalue problem:

$$
\frac{1}{\lambda_{R, N}}=\max \left\{\sum_{|\alpha| \leq N} \sum_{|\beta| \leq N} \mathcal{K}_{R, \alpha, \beta} c_{\alpha} \overline{c_{\beta}}: \sum_{|\alpha| \leq N}\left|c_{\alpha}\right|^{2}=1\right\} .
$$

We also let $\mathcal{K}_{R, N}=\left(\mathcal{K}_{\alpha, \beta}\right)_{|\alpha|,|\beta| \leq N}$ be a truncation of the matrix $\mathcal{K}_{R}$. Note that since

$$
\int_{\theta \in[0,2 \pi]^{d}}\left|\sum_{|\alpha| \leq N} c_{\alpha} P_{R, \alpha}\left(e^{i \theta}\right)\right|^{2} \frac{d \theta}{(2 \pi)^{d}}=\sum_{|\alpha|,|\beta| \leq N} c_{\alpha} \overline{c_{\beta}} \mathcal{K}_{\alpha, \beta}>0
$$

when $p(z)=\sum_{|\alpha| \leq N} c_{\alpha} P_{R, \alpha}(z)$ is not the zero polynomial, the matrix $\mathcal{K}_{R, N}$ is positive definite.

$(\Rightarrow)$ Suppose a reproducing kernel exists, i.e.

$$
\sum_{\alpha \in \mathbb{N}_{0}^{d}}\left|P_{\alpha}(z)\right|^{2}
$$

is uniformly bounded on compact subsets of $\mathbb{C}^{d}$. In particular, for $z$ on the torus of radius $R$, there is some $M<\infty$ such that $\sum_{\alpha \in \mathbb{N}_{0}^{d}}\left|P_{\alpha}(z)\right|^{2} \leq M$. If we consider the $R$-scaling of the sequence and the resulting orthonormal polynomials, this implies that

$$
\sum_{\alpha \in \mathbb{N}_{0}^{d}}\left|P_{R, \alpha}(z)\right|^{2} \leq M
$$

for all $z$ on the unit torus. Note that the $N^{\text {th }}$ partial sum is equal to the trace of $\mathcal{K}_{R, N}$. Since $\mathcal{K}_{R, N}$ is positive, the trace is greater than the largest eigenvalue. Thus

$$
\begin{aligned}
\frac{1}{\lambda_{R, N}} & \leq \operatorname{tr}\left(K_{R, N}\right) \\
& =\sum_{|\alpha| \leq N}\left(\int_{\theta \in[0,2 \pi]^{d}}\left|P_{R, \alpha}\left(e^{i \theta}\right)\right|^{2} \frac{d \theta}{(2 \pi)^{d}}\right) \\
& =\int_{\theta \in[0,2 \pi]^{d}}\left(\sum_{|\alpha| \leq N}\left|P_{R, \alpha}\left(e^{i \theta}\right)\right|^{2}\right) \frac{d \theta}{(2 \pi)^{d}} \\
& \leq \int_{\theta \in[0,2 \pi]^{d}} M \frac{d \theta}{(2 \pi)^{d}} \\
& =M<\infty .
\end{aligned}
$$

Hence $\lambda_{R, N} \geq \frac{1}{M}$ for all $N$, and thus is bounded away from zero as $N \rightarrow \infty$.

$(\Leftarrow)$ Suppose $\lambda_{R, N} \rightarrow \gamma_{R}>0$ as $N \rightarrow \infty$. From this we conclude that the largest eigenvalue of $\mathcal{K}_{R, N}$ is bounded by $\frac{1}{\gamma_{R}}$ for all $N$, and since it is positive, $\left\|\mathcal{K}_{R, N}\right\|$ is also bounded by $\frac{1}{\gamma_{R}}$. We wish to show that for each compact $E \subseteq \mathbb{C}^{d}$, there is some $M<\infty$ so that $\sum_{\alpha \in \mathbb{N}_{0}^{d}}\left|P_{\alpha}(z)\right|^{2}<M$ for all $z \in E$.

For an arbitary set of complex numbers $\left\{c_{\alpha}\right\}_{|\alpha| \leq N}$, the boundedness of the operator $\mathcal{K}_{R, N}$ implies

$$
\sum_{|\alpha|,|\beta| \leq N} c_{\alpha} \overline{c_{\beta}} \mathcal{K}_{R, \alpha, \beta} \leq \frac{1}{\gamma_{R}} \sum_{|\alpha| \leq N}\left|c_{\alpha}\right|^{2},
$$


and by letting $p(z)=\sum_{|\alpha| \leq N} c_{\alpha} P_{R, \alpha}(z)$, this is equivalent to

$$
\int_{\theta \in[0,2 \pi]^{d}}\left|p\left(e^{i \theta}\right)\right|^{2} \frac{d \theta}{(2 \pi)^{d}} \leq \frac{1}{\gamma_{R}} L_{R}\left(|p|^{2}\right) .
$$

Now let $E$ be a compact set in $\mathbb{C}^{d}$ and let $R>0$ be a real number such that $E$ is a subset of the open polydisk $D(0, R)^{d}$. Then the set $E_{R}:=\frac{1}{R} E$ is contained in the unit polydisk, and if $p$ is a polynomial, then

$$
p(z)=p_{R}\left(\frac{z}{R}\right)
$$

so values taken by $p(z)$ on $E$ are the same as values taken by $p_{R}(z)$ on $E_{R}$.

Let $y \in E_{R}$. By Cauchy's integral formula,

$$
p_{R}(y)=\frac{1}{(2 \pi)^{d}} \int_{[0,2 \pi]^{d}} \frac{p_{R}\left(e^{i \theta}\right)}{\left(y_{1}-e^{i \theta_{1}}\right)\left(y_{2}-e^{i \theta_{2}}\right) \cdots\left(y_{d}-e^{i \theta_{d}}\right)} e^{i\left(\theta_{1}+\theta_{2}+\cdots+\theta_{d}\right)} d \theta .
$$

Using Hölder's inequality, we obtain

$$
\left|p_{R}(y)\right|^{2} \leq \int_{[\theta \in 2 \pi]^{d}}\left|p_{R}\left(e^{i \theta}\right)\right|^{2} \frac{d \theta}{(2 \pi)^{d}} \cdot \int_{\theta \in[0,2 \pi]^{d}} \frac{1}{\left|y_{1}-e^{i \theta_{1}}\right|^{2} \cdots\left|y_{d}-e^{i \theta_{d}}\right|^{2}} \frac{d \theta}{(2 \pi)^{d}} .
$$

Letting

$$
M=\max \left\{\frac{1}{\gamma_{R}} \int_{\theta \in[0,2 \pi]^{d}} \frac{1}{\left|w_{1}-e^{i \theta_{1}}\right|^{2} \cdots\left|w_{d}-e^{i \theta_{d}}\right|^{2}} \frac{d \theta}{(2 \pi)^{d}}: w \in E_{R}\right\},
$$

we combine this with (2.1), so for any polynomial $p_{R}$,

$$
\left|p_{R}(y)\right|^{2} \leq M L_{R}\left(\left|p_{R}(x)\right|^{2}\right)
$$

Now we pick the particular polynomial $p_{R}(z)=\sum_{|\alpha| \leq N} \overline{P_{R, \alpha}(y)} P_{R, \alpha}(z)$ and apply the inequality

$$
\left.\left.\left|\sum_{|\alpha| \leq N}\right| P_{R, \alpha}(y)\right|^{2}\right|^{2} \leq M \sum_{|\alpha| \leq N}\left|P_{R, \alpha}(y)\right|^{2},
$$

which becomes

$$
\sum_{|\alpha| \leq N}\left|P_{R, \alpha}(y)\right|^{2} \leq M
$$

Since there is a uniform bound over all $N$, this implies

$$
\sum_{\alpha \in \mathbb{N}_{0}^{d}}\left|P_{R, \alpha}(y)\right|^{2} \leq M
$$

Thus the sum $\sum_{\alpha}\left|P_{R, \alpha}(z)\right|^{2}$ is bounded uniformly in $\frac{1}{R} E$ which implies that the sum $\sum_{\alpha}\left|P_{\alpha}(z)\right|^{2}$ is bounded uniformly by $M$ in $E$, and thus such a reproducing kernel exists.

This theorem along with some considerations from the one variable case gives the proof of Theorem 1.1. In this case $\left\{s_{n}\right\}$ is a positive multisequence exactly when there is a positive measure $\mu$ which represents $L$ in the sense that

$$
L(p)=\int_{\mathbb{R}} p(x) d \mu .
$$

In one dimension it is known that the sum $\sum_{n=0}^{\infty}\left|P_{n}(z)\right|^{2}<\infty$ for some $z \in \mathbb{C} \backslash \mathbb{R}$ if and only if the sum converges uniformly on compact subsets of $\mathbb{C}$. Also in this 
case, this sum converges if and only if the measure $\mu$ associated with the moment sequence is indeterminate (see [1]).

So assuming that $\mu$ on $\mathbb{R}$ is indeterminate, then there exists a reproducing kernel for the Hilbert space of polynomials implying that $\sum_{n=0}^{\infty}\left|P_{n}(z)\right|^{2}$ converges uniformly on compact subsets of $\mathbb{C}$. By Theorem 2.1 this leads us to the conclusion that $\gamma_{1}>0$, i.e. the smallest eigenvalues of $H_{N}$ are uniformly bounded away from 0 .

Now assuming that $\gamma_{1}>0$, the proof of Theorem 2.1 implies that the sum $\sum_{n=0}^{\infty}\left|P_{n}(z)\right|^{2}$ is bounded uniformly on compact subsets of the open unit disk. Thus there is some $z_{0} \in \mathbb{D} \backslash \mathbb{R}$ so that $\sum_{n=0}^{\infty}\left|P_{n}\left(z_{0}\right)\right|^{2}<\infty$, hence the measure $\mu$ is indeterminate.

\section{Application}

We would like to know more about $\gamma_{R}$. If we consider it as a function $\gamma$ : $(0, \infty) \rightarrow[0, \infty)$ given by $\gamma(R)=\gamma_{R}$, then Theorem 2.1 implies that if $\gamma(R)>0$, then $\gamma(S)>0$ for all $S<R$. We see this since $\gamma(R)>0$ implies that a reproducing kernel for $\mathcal{P}$ exists and converges uniformly on compact subsets of the polydisk of radius $R$. Then in the converse portion of the argument, the fact that a reproducing kernel exists which converges uniformly on the torus of radius $S$ is used to show that $\lambda_{S, N}$ are uniformly bounded away from zero as $N \rightarrow \infty$, hence $\gamma(S)>0$.

If a multisequence in any number of variables can be represented by a measure $\sigma$, then $\sigma$ is indeterminate if and only if every $R$-scaling $\sigma_{R}$ is as well. In one variable, Berg, Chen, and Ismail's result implies that $\gamma_{1}>0 \Leftrightarrow \gamma_{R}>0$ for some $R>0$, which is equivalent to $\gamma_{S}>0$ for every $S>0$. This is an open question whether this property holds for multisequences in multiple variables, but in Theorem 3.1 we establish a class of multisequences which do possess this property.

We would like to extend more of Theorem 1.1 to the $d>1$ case. Presently we have that the conditions

$$
\begin{aligned}
& \gamma_{1}>0, \\
& \gamma_{R}>0, \text { for all } R>0, \\
& \sum_{\alpha \in \mathbb{N}_{0}^{d}}\left|P_{\alpha}(z)\right|^{2} \text { is bounded uniformly on compact sets, } \\
& \sum_{\alpha \in \mathbb{N}_{0}^{d}}\left|P_{\alpha}(z)\right|^{2}<\infty \text { for some } z \in(\mathbb{C} \backslash \mathbb{R})^{d},
\end{aligned}
$$

are related by the implications

$$
(3.1) \Leftarrow(3.2) \Leftrightarrow(3.3) \Rightarrow(3.4),
$$

and also (3.1) $\Rightarrow(3.4)$ if we pick some $z$ contained in the open unit polydisk. We would like also to have (3.1) $\Rightarrow(3.2)$ and (3.4) $\Rightarrow(3.3)$ in $d>1$ as it is in $d=1$. We show this is the case for a certain class of multisequences.

Theorem 3.1. Let $\left\{s_{\alpha}\right\}$ be a positive multisequence which satisfies the condition

$$
s_{\alpha}=s_{\left(\alpha_{1}, 0,0, \ldots, 0\right)} s_{\left(0, \alpha_{2}, 0, \ldots, 0\right)} \cdots s_{\left(0, \ldots, 0, \alpha_{d}\right)} .
$$

Then there is a positive measure $\mu$ on $\mathbb{R}^{d}$ which represents the multisequence in the sense that

$$
\int_{\mathbb{R}^{d}} x^{\alpha} d \mu=s_{\alpha}
$$


the conditions (3.1), (3.2), (3.3), and (3.4) are equivalent; and these conditions all imply indeterminacy of $\mu$.

Proof. Let $\left\{s_{\alpha}\right\}$ satisfy this condition and let $L$ be the functional derived from the multisequence. We define a collection of $d$ sequences by for each $j=1, \ldots, d$, and let

$$
s_{j, n}=s_{(0, \ldots, 0, n, 0, \ldots, 0)},
$$

where the $n$ is in the $j^{\text {th }}$ place. The positivity of the sequence $s_{j, n}$ follows from the positivity of the multisequence $\left\{s_{\alpha}\right\}$, so for each $1 \leq j \leq d$, the theorem of Hamburger (1] p.30) implies that there exists a positive measure $\mu_{j}$ on $\mathbb{R}$ for which

$$
\int_{\mathbb{R}} x^{n} d \mu_{j}(x)=s_{j, n} .
$$

If we define a measure $\mu:=\mu_{1} \otimes \mu_{2} \otimes \cdots \otimes \mu_{d}$ on $\mathbb{R}^{d}$, the multiplicative condition (3.5) implies the first assertion, that

$$
\int_{\mathbb{R}^{d}} x^{\alpha} d \mu=\left(\int_{\mathbb{R}} x_{1}^{\alpha_{1}} d \mu_{1}\right) \cdots\left(\int_{\mathbb{R}} x_{d}^{\alpha_{d}} d \mu_{d}\right)=s_{1, \alpha_{1}} s_{2, \alpha_{2}} \cdots s_{d, \alpha_{d}}=s_{\alpha}
$$

for each monomial $x^{\alpha}$.

For the measure $\mu$, we construct a suitable set of orthonormal polynomials $\left\{P_{\alpha}(x)\right\}$. For each $1 \leq j \leq d$, let $\left\{P_{j, n}(x)\right\}_{n=0}^{\infty}$ be the standard set of orthonormal polynomials associated with the measure $\mu_{j}$ in $\mathbb{R}(1]$ p. 3$)$. If we define for each $\alpha \in \mathbb{N}_{0}^{d}$ the polynomial

$$
P_{\alpha}(x):=P_{1, \alpha_{1}}\left(x_{1}\right) P_{2, \alpha_{2}}\left(x_{2}\right) \cdots P_{d, \alpha_{d}}\left(x_{d}\right),
$$

then the resulting set of polynomials $\left\{P_{\alpha}(x)\right\}$ span $\mathbb{C}[x]$ and satisfy the orthonormal property

$$
\int_{\mathbb{R}^{d}} P_{\alpha}(x) \overline{P_{\beta}(x)} d \mu(x)=\delta_{\alpha_{1}, \beta_{1}} \delta_{\alpha_{2}, \beta_{2}} \cdots \delta_{\alpha_{d}, \beta_{d}}=\delta_{\alpha, \beta} .
$$

Now for any $z \in \mathbb{C}^{d}$ and $N \in \mathbb{N}$, we consider the sum

$$
\begin{aligned}
\sum_{\substack{\left|\alpha_{j}\right| \leq N \\
1 \leq j \leq d}}\left|P_{\alpha}(z)\right|^{2}=\left(\sum_{\alpha_{1}=0}^{N}\left|P_{1, \alpha_{1}}\left(z_{1}\right)\right|^{2}\right) & \left(\sum_{\alpha_{2}=0}^{N}\left|P_{2, \alpha_{2}}\left(z_{2}\right)\right|^{2}\right) \\
& \ldots\left(\sum_{\alpha_{d}=0}^{N}\left|P_{d, \alpha_{d}}\left(z_{d}\right)\right|^{2}\right) .
\end{aligned}
$$

This sum converges as $N \rightarrow \infty$ if and only if each of the sums on the right hand side converges. We will show that for this measure, (3.1) $\Rightarrow$ (3.4) for a generic $z \in$ $(\mathbb{C} \backslash \mathbb{R})^{d}$ and $(3.4) \Rightarrow(3.3)$. In the process we prove that these imply indeterminacy of $\mu$.

$(\underline{3.10}) \Rightarrow(3.4))$. Suppose $\gamma_{1}>0$. Define

$$
J_{N}^{j}=\left(s_{(0, \ldots, 0, k+l, 0, \ldots, 0)}\right)_{k, l=0}^{N},
$$

where the $k+l$ is in the $j^{\text {th }}$ coordinate, to be the $(N+1) \times(N+1)$ truncated Hankel matrix associated with the measure $\mu_{j}$, and let $\eta_{N}^{j}$ be its smallest eigenvalue. Then 
$\eta_{N}^{j}>\gamma_{1}$ for each $j$ and $N \in \mathbb{N}$ since $J_{N}^{j}$ is a compression of the operator $H_{N}$. By Theorem 1.1 it follows that $\mu_{j}$ is indeterminate and

$$
\sum_{\alpha_{j}=0}^{\infty}\left|P_{j, \alpha_{j}}\left(z_{j}\right)\right|^{2}
$$

converges for some $z_{j} \in \mathbb{C} \backslash \mathbb{R}$. If $z=\left(z_{1}, z_{2}, \ldots, z_{d}\right) \in(\mathbb{C} \backslash \mathbb{R})^{d}$, then (3.4) follows from (3.6).

$(\underline{(3.4)} \Rightarrow(\sqrt{3.31}))$. Now let $z \in(\mathbb{C} \backslash \mathbb{R})^{d}$ be a point such that $\sum_{\alpha \in \mathbb{N}_{0}^{d}}\left|P_{\alpha}(z)\right|^{2}<\infty$. Via (3.6), this implies that $\sum_{\alpha_{j}=0}^{\infty}\left|P_{j, \alpha_{j}}\left(z_{j}\right)\right|^{2}<\infty$ for each $1 \leq j \leq d$, which implies that $\mu_{j}$ is indeterminate. Then $\sum_{\alpha_{j}=0}^{\infty}\left|P_{j, \alpha_{j}}\left(z_{j}\right)\right|^{2}$ converges uniformly on compact subsets of $\mathbb{C}$. Let $E$ be a compact subset of $\mathbb{C}^{d}$ and $L \geq 0$ so that $E \subseteq(\overline{\mathbb{D}(0, L)})^{d}$. Then $(\underline{3.6})$ tells us that $\sum_{\alpha \in \mathbb{N}_{0}^{d}}\left|P_{\alpha}(z)\right|^{2}$ converges uniformly on $(\overline{\mathbb{D}(0, L)})^{d}$ and thus on $E$.

We now have that (3.1), (3.2), (3.3), and (3.4) are equivalent when (3.5) applies, and our arguments have shown that these conditions imply indeterminacy for each $\mu_{j}$. Since at least one of these measures is indeterminate, Petersen's theorem leads to indeterminacy of $\mu$.

This theorem provides that when a positive multisequence is multiplicative in this sense, a solution to the associated moment problem exists, and the existence of a reproducing kernel implies indeterminacy. In the case of deciding indeterminacy of an arbitrary moment multisequence, the role of reproducing kernels is not yet fully clear. Fuglede's introduction of the notions of strong determinacy and ultradeterminacy [5] and the creation of new indeterminate moment problems from old ones [7] provide ground for further study, and we will address these in a future article.

\section{REFERENCES}

1. Akhiezer, N.I. The Classical Moment Problem and Some Related Questions in Analysis, Oliver and Boyd, Edinburgh, 1965.

2. Berg, C. Chen, Y. and Ismail, M. Small eigenvalues of large Hankel matrices: the indeterminate case, Math. Scand. 91 (2002), 67-81. MR1917682(2003f:47042)

3. Berg, C. Christensen, J. P. R. and Jensen, C. U., A Remark on the Multidimensional Moment Problem, Math. Ann. 243 (1979), 163-169. MR543726 (81e:44008)

4. Dunkl, C. F. And Xu, Y. Orthogonal Polynomials of Several Variables, Cambridge University Press, Cambridge, 2001. MR 1827871(2002m:33001)

5. Fuglede, B. The Multidimensional Moment Problem, Expo. Math. 1 (1983), 47-65. MR693807 (85g:44010)

6. Petersen, L. C. On the Relation Between the Multidimensional Moment Problem and the One-Dimensional Moment Problem, Math. Scand. 51 (1982), 361-366. MR690537 (84g:44005)

7. Putinar, M. and Scheiderer, C. Multivariate moment problems: Geometry and indeterminateness, Ann. Scuola Norm. Sup. Pisa Cl. Sci. (5) Vol. V (2006), 137-157. MR2244695

8. Saitoh, S., Theory of Reproducing Kernels and Its Applications, Longman Scientific and Technical, Harlow Essex, 1988. MR 983117 (90f:46045)

9. Schmüdgen, K., An Example of a Positive Polynomial which is not a Sum of Squares of Polynomials; A Positive, but not Strongly Positive Functional, Math. Nachr. 88 (1979), 385390. MR 543417 (81b:12024)

Department of Mathematics, California State University, Channel Islands, One University Drive, Camarillo, California 93012

E-mail address: roger.roybal@csuci.edu 